\title{
A Blur Restoration Research on Sequential Images of Bullets Taken in the Penetration Test
}

\author{
Bin Chen \\ College of Basic Education for Officers, National University of Defense Technology \\ Changsha, China, 410072 \\ email:chenbin_gfkd@sina.com
}

\begin{abstract}
In order to remove the motion blur in sequential images of bullets taken in the penetration test, firstly we found out the relationship among the degradation model, direction of motion and blurred length of a bullet image in this paper. Then the radon transformation was used to found out the direction of motion. At the same time, the blurred length was got by using differential autocorrelation method. Finally, we accomplished the restoration of the bullet by using the constrained least squares filter. The result shows that the method taken in this paper is simple and feasible. And the result of the restoration is very satisfying.
\end{abstract}

Keywords-motion blur; direction of motion; blurred length; radon transformation; differential autocorrelation; constrained least squares

\section{INTRODUCTION}

In the penetration test, there was a kind of relative motion between the bullet and high-speed camera. The relative motion led to blur and degeneration of the collected bullet image, which brought relatively large error for the subsequent target extraction and parameter measurement. Although some researchers have done much relative work [1, 3, 4], the ideal result has not been obtained yet. In order to restore the degraded bullet image, we need to definitude the degradation mechanism and process of them, and then conduct an inverse operation to get the estimation of the original image under some optimal sense. During very short exposure time, the flight status of the bullet could be approximated seen as uniform linear motion. According to the characteristics of the image, we can identify the fuzzy parameter, obtain the point spread function and remove the smear produced by movement of the bullet.

\section{A. Degradation model of the bullet image}

The motion distances of the image $(f(x, y))$ in direction $\mathrm{x}$ and $\mathrm{y}$ are supposed to be $\mathrm{x}_{0}(\mathrm{t})$ and $\mathrm{y}_{0}(\mathrm{t})$ during the exposure time. The total exposure time is $\mathrm{T}$. The total exposure of the recording medium is the integration of the time the shutter from open to close. According to the assumptions above, the blurred image can be denoted as follow:

$$
g(x, y)=\int_{0}^{z} t\left[x-x_{0}(t), y-y_{0}(t)\right] d t
$$

The motion state of the bullet in the image to be taken is uniform linear motion. The blurred length of the bullet image can be remarked as $\mathrm{L}$ during the exposure time $(\mathrm{T})$. The inclined angle between the direction of motion blur and $\mathrm{x}$-axis is $\theta$. The blurred length generated by the image in the direction of $x$ is $a(a=L \cos \theta)$, and the blurred length generated by the image in the direction of $y$ is $b(b=L \sin \theta)$. The spatial domain expression of the degradation model of the bullet image can be remarked as follow:

$$
\begin{aligned}
& \Xi(x, y)-\int_{0}^{T} f\left(x-\frac{a t}{T}, y-\frac{\partial t}{T}\right) d t \\
= & \int_{0}^{T} f\left(x-\frac{t E \cos \theta}{T}, y-\frac{t \varepsilon \sin \theta}{T}\right) d t
\end{aligned}
$$

If taking the Fourier transformation to (2), and supposing

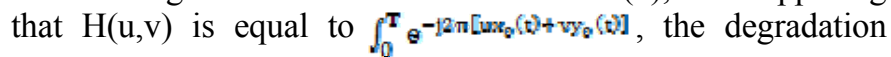
model's frequency expression of the bullet image can be obtained as follow:

$$
G(u, v)=F(u, v) H(u, v)
$$

In (3), G(u,v), F(u,v) and H(u,v) correspond to the Fourier transformation of blurred image $(\mathrm{g}(\mathrm{x}, \mathrm{y}))$, original image $(\mathrm{f}(\mathrm{x}, \mathrm{y}))$ and point spread function $(\mathrm{h}(\mathrm{x}, \mathrm{y}))$ respectively.

$\mathrm{H}(\mathrm{u}, \mathrm{v})$ is also called transfer function. After transforming $\mathrm{H}(\mathrm{u}, \mathrm{v})$ with the Fourier transformation, the blur and degeneration's point spread function caused by the bullet's uniform motion can be got as follow:

$$
h(x, y)=\left\{\begin{array}{cc}
\frac{1}{L} & 0 \leq x \leq L \cos \theta, 0 \leq y \leq x \tan \theta \\
0 & \text { else }
\end{array}\right.
$$

From the degradation model of the bullet image, we can see that if the direction of motion $(\theta)$ and blurred length (L) are identified, the transfer function of motion blur can be got and then the image restoration can be taken by a classical method.

\section{IDENTIFICATION OF THE MOTION'S DIRECTION}

\section{A. Spectral characteristics of the motion blurred image}

In order to reserve generality, if the motion blur's direction is taken as horizontal direction, the formula of the blur and degeneration's point spread function becomes as follow:

$$
h(x, y)=\frac{1}{2} \quad 0 \leqslant x<L_{n} y=0
$$


The size of $h(x, y)$ can be extended by zero padding until it becomes the same size as $\mathrm{f}(\mathrm{x}, \mathrm{y})$, and then, transform it with discrete Fourier transformation. Finally, we can get the formula as follow:

$$
H(u, v)=\sum_{x=0}^{L-1} \frac{1}{L} e^{-j 2 \pi \frac{w x}{N}}
$$

The formula above indicates that $\mathrm{H}(\mathrm{u}, \mathrm{v})$ has nothing to do with $\mathrm{v}$, so we can just take $\mathrm{u}$ into consideration. When $\mathrm{u}=0$, $\mathrm{H}(\mathrm{u})=1$. When, $\mathrm{u} \neq 0$, the formula as follow can be got:

$$
H(u)=\sum_{x=0}^{L}=\frac{1}{L} e^{-f 2 \pi \frac{w x}{X}}=\frac{1}{L} \times \frac{i-e^{-j n \pi \frac{u z}{X}}}{1-e^{-j \ln \frac{\pi}{X}}}
$$

In (7), $u \in[0, N-1] . H(0)=1$, so $H(u)$ has no climax. When $u$ is equal to integer multiples of $\mathrm{N}, \mathrm{H}(\mathrm{u})=0$. That is to say, $\mathrm{H}(\mathrm{u})$ will appear to be zero every $\mathrm{NL}$ points. There are (L-1) distribute zero points in all. Because the degraded image is the result of the convolution between the degenerate function and original image, zero points also appear in the spectrum of the degraded image. The spectrum of the degraded image performs as uniformly-spaced dark fringes. According to the timefrequency characteristics of Fourier transformation, we can know that the dark stripes and movement direction are perpendicular to each other. As long as we are able to detect the dark stripes in the spectrum of the bullet's degraded image, the direction of the bullet's motion can be identified.

\section{B. Identification of the direction of motion based on radon transformation}

When the image is transformed with Radon transformation, the transformed values reflect the projection properties of the image in different directions. Because the basic idea is a duality between point and line, a line $(\rho=x \cos \theta+y \sin \theta)$ in the twodimensional space is mapped to a point $(R(\rho, \theta))$ in Radon space. Before transformation, the image is in the image space, and after transformation, the image is in the parameter space.

The linear Radon transformation of a continuous image can be defined as follow:

$$
R(\rho, \theta)=\iint_{D} f(x, y) \&(\rho-x \cos \theta-y \sin \theta) d x d y
$$

$\rho$ is equal to the distance between the origin of coordinates in the two-dimensional space and the line. $\theta(\theta \in[0, \pi])$ is equal to the included angle between the vertical line which starts from origin of the image to the line and $\mathrm{x}$-axis. The characteristic function $(\delta)$ is Dirac function. This function forces the integration to work along the line $\rho$.

If there are relatively bright or dark lines in the image, the corresponding parameter position after Radon transformation will be a peak (Bright lines are positive peaks, dark lines are negative lines). By detecting peak points, the positions and directions of lines in the image can be obtained. That is to say, the direction of the degraded image's motion can be identified by detecting lines in the frequency domain of the degraded image. In the detection of the lines, Radon transformation is used.

\section{IDENTIFICATION OF THE FUZZY LENGTH}

Because the motion state of the bullet is uniform linear motion, and its fuzzy and degraded Point spread function supports rectangular function whose support region is equal to L, the fuzzy function's differential autocorrelation in the direction of motion appears to be a peak value when i equals to 0 and valley value when $i$ equals to $\pm(L-1)$. The result is shown in Fig. 1.

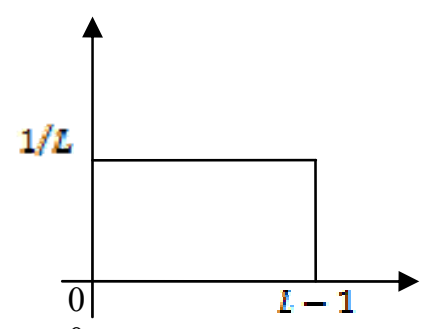

(a) Fuzzy function of uniform motion

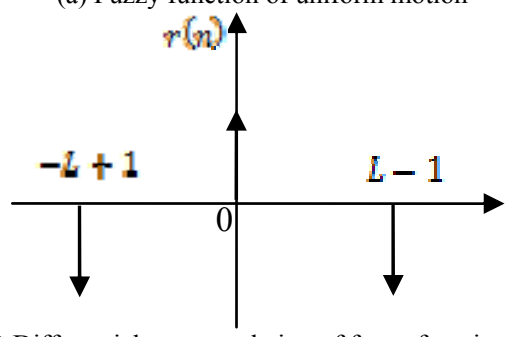

(b) Differential autocorrelation of fuzzy function

Fig. 1. Motion blur degenerate function's characteristic figure

The first-order difference operation of bullet's degraded image is done in the fuzzy direction of motion. The difference matrix can be regarded as $\mathrm{D}(\mathrm{x}, \mathrm{y})$. The differenced image can be regarded as $\mathrm{M}(\mathrm{x}, \mathrm{y})$, so the result can be got as follow:

$$
\begin{aligned}
& M(x, y)=g(x, y) * D(x, y) \\
& =f(x, y) * h(x, y) * D(x, y)
\end{aligned}
$$

$\mathrm{p}(\mathrm{x}, \mathrm{y})$ is regarded as $\mathrm{h}(\mathrm{x}, \mathrm{y}) * \mathrm{D}(\mathrm{x}, \mathrm{y})$. Because the motion blur is one dimensional, the direction of motion can be supposed to be horizontal (other directions can be revolved to horizontal position), so we can get the result as follow:

$$
M(x)=f(x) * p(x)
$$

The differenced image's self-correlation can be obtained, and it can be expressed as follow:

$$
\begin{aligned}
M(x) * M(-x) & =[f(x) * p(x)] *[f(-x) * p(-x)] \\
& =[f(x) * f(-x)] *[p(x) * p(-x)]
\end{aligned}
$$


In $(10), f(x) * f(-x)$ whose shape is shown in Fig. 4(a) is the image's self-correlation function, $\mathrm{p}(\mathrm{x})^{*} \mathrm{p}(-\mathrm{x})$ whose selfcorrelation shape is shown in Fig. 3(b) is motion blur degenerate function's first difference autocorrelation, so the result we get by operating based on the formula above should have the shape of Fig. 2(b).

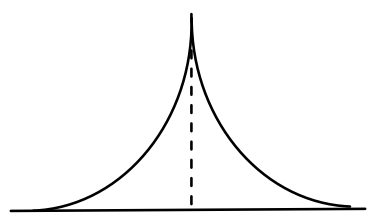

(a) Self-correlation function of the image (b) Differenced degraded image's self-correlation function

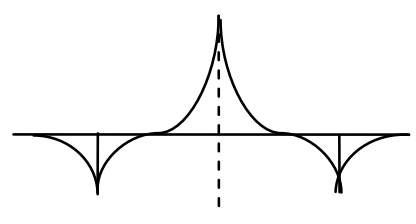

Fig. 2. The characteristics of the image's self-correlation function

So, the difference autocorrelation function of degraded image and the fuzzy function will appear the same maximum value at $x=0$, the same minimum value at $x= \pm L$. Blurred length $\mathrm{L}$ is half of the distance between two valley point According to the characteristic, blurred length can be identified.

Blurred length's specific identification steps can be conducted as the flow chart below.

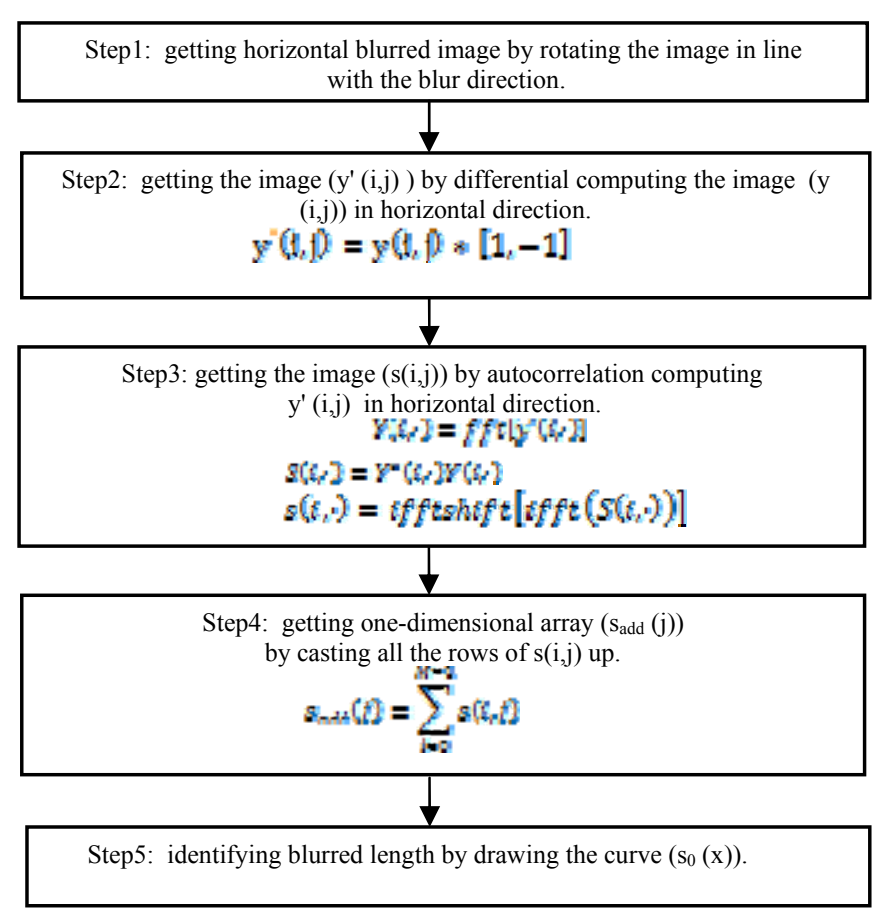

Fig. 3. The flow chart of the blurred length's identification

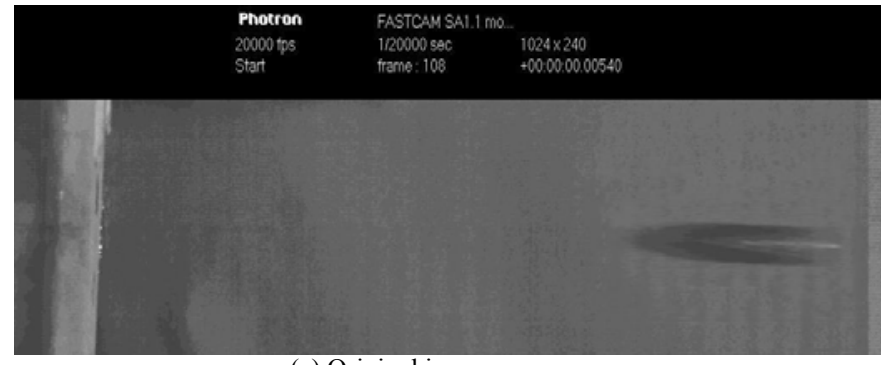

(a) Original image

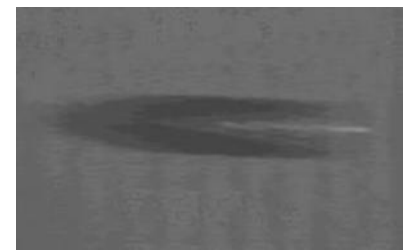

(b) Fuzzy region

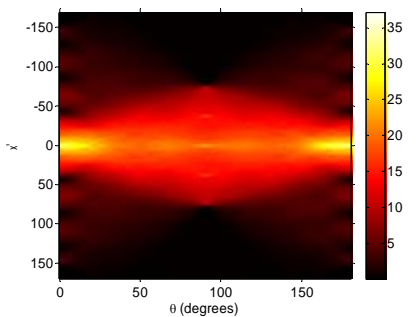

(d) Result of Radon transformation

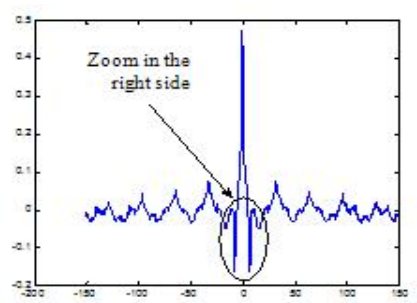

(f)Corresponding differential autocorrelation discrimination curve

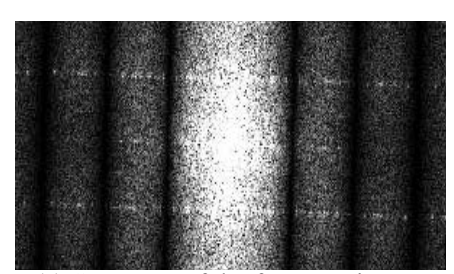

(c) Spectrum of the fuzzy region

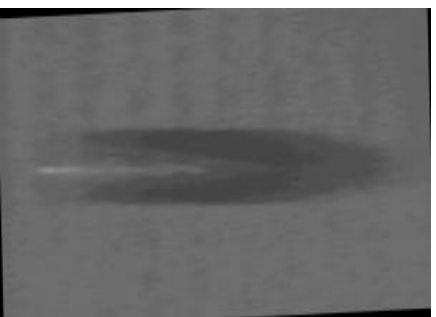

(e) Rotated image

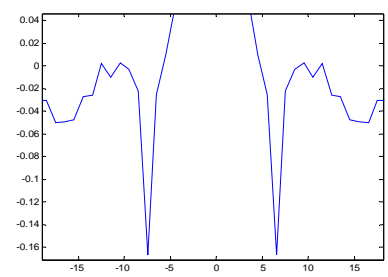

(g) Discrimination curve's drawing of partial enlargement

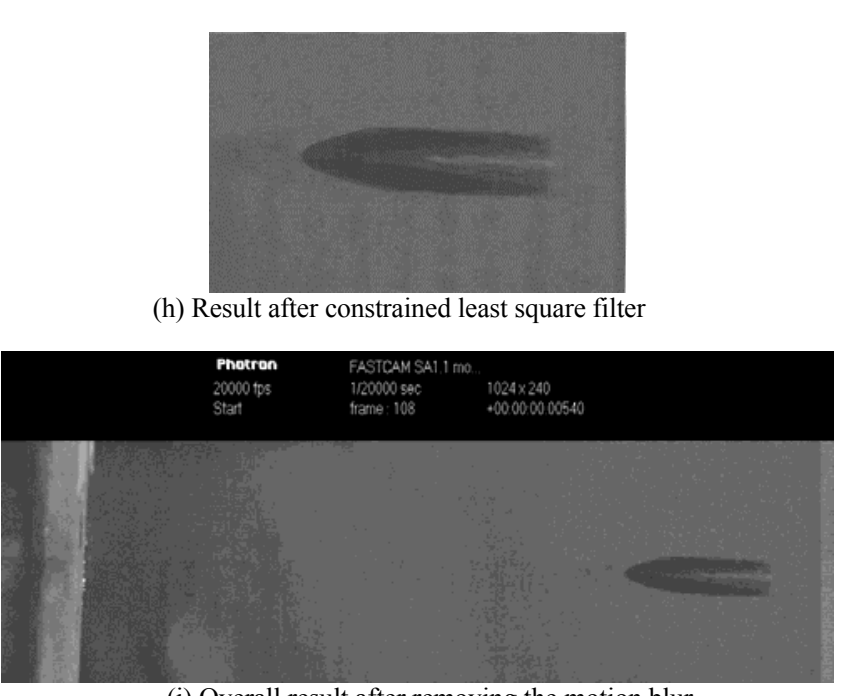

(i) Overall result after removing the motion blur

Fig. 4. The bullet image's effect after removing blur and restoring 


\section{RESTORATION OF THE BULlET'S MOTION BLUR}

After identifying the fuzzy parameters, it is easy to get the degenerate function's expression. The bullet's degraded image can be restored with a constrained least square filter algorithm. The sequential images of bullets can be restored by taking the method mentioned before. Because the motion blur and degradation only appear in the place of the bullet and its nearby place, we can just operate the corresponding pixel points.

Fig. 4 shows the working sketches of whole treating processes. Fig. 4(a) is the original image after grey enhancement. There is motion blur in Fig. 4(a). Fig. 4(b) is the locked bullet and its nearby place. Fig. 4(c) is the corresponding spectrogram. There are alternately dark and bright stripes in the corresponding spectrogram which conforms to the characteristic of motion blur's spectrogram. Fig. 4(d) is the result of the image that was transformed with Radon transformation by step of $0.05^{\circ}$. The detected blur direction is $177.95^{\circ}$. Fig. 4(e) is the image that was rotated after the blur direction being detected. The result shows that the rotated bullet in the image is horizontal. The result which inosculates with the actual situation indicates that the blur direction is the same as the bullet's axis direction. Fig. 4(f) is the corresponding differential autocorrelation discrimination curve. Fig. 4(g) is the discrimination curve's drawing of partial enlargement. The detected blurred length is 7 pixels. Fig. 4(h) is the result of the image that was dealt with constrained least square filter. The shadows in the head and tail of the bullet were removed after the operation of filtering. Fig. 4(i) is the final result by covering the original image with dealt pixels.
Comparing Fig. 4(i) and Fig. 4(a), we can see that shadow of the recovered bullet was removed, and the bullet's length became normal. The image of the toll bullet is clearer, and it is easy to extract the bullet in high precision.

\section{CONCLUSION}

The research work done in this paper is described as followed. Firstly, taking advantage of the feature that there are dark stripes in the bullet's degraded image spectrum, the direction of motion is detected by Radon transform. Secondly, the blurred length is identified according to the feature that there are symmetrical valley points in the bullet's degraded image's corresponding differential autocorrelation. Finally, by using the constrained least squares filter algorithm, the smear in the bullet's image is removed and the bullet restoration is realized. This work is of important significance to the experimental research about impact and penetration field.

\section{REFERENCES}

[1] C.Y. Dai, "Research on image restoration method for motion blur", Zhejiang University Master's Dissertation, 2011

[2] Q.Y. Yuan, Basic for digital image processing, Beijing, Tsinghua University Press, 2009

[3] X. Le, J. Cheng, and M. Li, "Improve approach to motion blur identification based on Radon transform", Infrared and Laser Eng. vol. 40, No. 5, pp. 963-969, 2011

[4] M. Hao, L. Fang, "Application of constrained least squares filtering on image restoration technology", Experiment. Sci. Technol. vol. 8, No. 5, pp. $21-23,2010$ 